Copyright (C) 2015 IEEE. Personal use of this material is permitted. Permission from IEEE must be obtained for all other uses, in any current or future media, including reprinting/republishing this material for advertising or promotional purposes, creating new collective works, for resale or redistribution to servers or lists, or reuse of any copyrighted component of this work in other works. 


\title{
On transmission of a continuous signal via a noiseless binary channel *
}

\author{
Nikolai Dokuchaev
}

December 29, 2014

\begin{abstract}
We study the problem of the transmission of currently observed time variable signals via a channel that is capable of sending a single binary signal only for each measurement of the underlying process. For encoding and decoding, we suggest a modification of the adaptive delta modulation algorithm. This modification ensures tracking of time variable signals. We obtained upper estimates for the error for the case of noiseless transmission.

Index Terms: encoding, communication bit-rate constraints, adaptive delta modulation, noiseless binary channel, A/D conversion
\end{abstract}

\section{Introduction}

We study the problem of transmission of a currently observed continuous time signal via a noiseless binary channel. The evolution law for the underlying continuous time signal is not supposed to be known; only some mild conditions on the signal regularity are imposed. In particular, the signal is not necessarily continuous, and unexpected jumps may occur. We consider the situation where the channel capacity is insufficient to send in real time sufficiently

${ }^{*}$ This is a pre-copy-editing, author-produced PDF of a paper accepted to IEEE Signal Processing Letters following peer review. The definitive publisher-authenticated version is available online at http://www.signalprocessingsociety.org/publications/periodicals/letters/

The author is with Department of Mathematics and Statistics, Curtin University, GPO Box U1987, Perth, Western Australia, 6845 (email N.Dokuchaev@curtin.edu.au).This work was supported by ARC grant of Australia DP120100928 to the author. 
accurate approximations of the current measurements. Therefore, the observed measurements have to be encoded, transmitted in the encoded form, and decoded. This problem may arise, for example, for remote control of underwater vehicles, since communication is severely limited underwater (see [1]).

The paper suggests a modification of the systems from $[2,3,4]$, where limited capacity digital channels were studied in stochastic setting. In [2], a related filtering problem was considered for the case of bounded random disturbances. A case of decreasing Gaussian disturbances was studied in [3] for a scalar system. In [4], a filtering problem was studied for the case of non-decreasing Gaussian disturbances for vector processes. The present paper considers an extreme case of a binary channel, i.e., one-bit capacity channel that can transmit a single binary signal for a single measurement of the underlying process. This channel connects two subsystems of a dynamical system. The first subsystem, that is called Encoder, receives the real-valued measurements and converts them into a binary symbolic sequence which is sent over the communication channel. For each measurement, only one single bit signal can be sent. The second subsystem (Decoder) receives this symbolic sequence and converts it into a real-valued state estimate. Therefore, the effectiveness of the entire system is defined by the effectiveness of the binary encoding algorithm. This encoding problem was widely studied in the literature. In non-causal setting, some important results can be found in $[5,6,8,9]$; see also the bibliography therein. In $[5,6,9]$, the encoding was studied in the framework of the sampling theory and Fourier analysis. In [8], a sequential binary estimator based on stochastic contamination was obtained for continuous processes.

For our particular task, we have restrictions of causality. To satisfy this condition, we suggest a modification of the adaptive delta modulation algorithm introduced by Jayant [10] for voice transmission; see more recent development in [11] and the bibliography therein. The suggested algorithm ensures stable tracking of time variable signals using just one bit for each measurement. The algorithm does not depend on the parameters of the evolution law and the distributions of the underlying process. We obtained the upper estimates of the error for the case of a noiseless transmission.

\section{Problem statement and the result}

Let $x(t)$ be a continuous time state process observed at times $t_{k}=k \delta, k=0,1,2, \ldots$, where $\delta>0$ is given. 
Suppose estimates of the current state $x(t)$ are required at a distant location, and are to be transmitted via a digital communication channel such that only one bit of data may be sent at each time $t_{k}$, i.e., a binary channel. For this task, we consider a system which consists of the encoder, the transmission channel, and the decoder. For each observation $x\left(t_{k}\right)$, the encoder produces a one-bit symbol $h_{k}$ which is transmitted via the channel and then received by the decoder; the decoder produces an estimate $\left.y(t)\right|_{\left[0, t_{k}\right]}$ which depends only on $h_{1}, \ldots ., h_{k}$. In other words, the process $x(t)$ is supposed to be sampled at times $t_{k}$, encoded, transmitted via the channel and then decoded. We assume that the transmission is noiseless.

It is important that, for each sample for each sampling point $t_{k}$, only one bit of information can be transmitted. The corresponding algorithm is suggested below.

Let real numbers $y_{0}, M_{0}>0, \bar{M}>0$, and $a \in(1,2]$ be given parameters that are known both to the encoder and the decoder. The algorithm can be described as follows.

1. Sample values $x\left(t_{k}\right)$ are taken;

2. The encoder computes a sequence $\left\{\left(y_{k}, M_{k}\right)\right\}_{k \geq 1} \subset \mathbf{R}^{2}$ and produces a sequence of binary symbols $\left\{h_{k}\right\}$ consequently for $k=-1,0,1,2, \ldots$ by the following rule: $h_{-1}=1$, and

$$
h_{k}= \begin{cases}1, & \text { if } \quad y_{k}<x\left(t_{k}\right) \\ -1, & \text { if } \quad y_{k}>x\left(t_{k}\right) \\ -h_{k-1}, & \text { if } \quad y_{k}=x\left(t_{k}\right)\end{cases}
$$

where

$$
\begin{gathered}
y_{k}=y_{k-1}+h_{k-1} M_{k-1} \delta, \quad k=1,2, \ldots \\
M_{k}=\left\{\begin{array}{l}
a M_{k-1}, \quad \text { if } k \notin \mathcal{T} \text { and } k-1 \notin \mathcal{T} \\
M_{k-1}, \quad \text { if } k \notin \mathcal{T} \text { and } k-1 \in \mathcal{T} \\
\max \left(a^{-1} M_{k-1}, \bar{M}\right), \quad \text { if } \quad k \in \mathcal{T},
\end{array}\right.
\end{gathered}
$$

and where $\mathcal{T}=\left\{k \geq 1: h_{k-1} h_{k}<0\right\}$.

3. The binary symbol $h_{k}$ is transmitted via the channel. 
4. The decoder computes the same sequence $\left\{\left(y_{k}, M_{k}\right)\right\}_{k \geq 1} \subset \mathbf{R}^{2}$ using the received values $\left\{h_{k}\right\}$ by the same rule as the encoder.

5. Finally, the decoder computes estimate $y(t)$ of the process $x(t)$ as

$$
y(t)=y_{k}+h_{k} M_{k}\left(t-t_{k}\right), \quad t \in\left[t_{k}, t_{k+1}\right],
$$

and where $k=0,1,2, \ldots, y(0)=y_{0}$.

Note that this algorithm represents a modification of the Jayant's adaptive delta modulation algorithm [10, 11], where it was assumed that, in our notations,

$$
\bar{M}=0, \quad M_{k}=a M_{k-1} \quad \text { if } \quad k-1 \in \mathcal{T} .
$$

The novelty of algorithm (1)-(4) is that it allows three possible values of $M_{k} / M_{k-1}$ on each step instead of two, and uses two bit memory instead of one. It was shown in [11] that Jayant's algorithm allows to track benchmark constant processes. We show below that he suggested changes ensure stable tracking processes for variable in time underlying processes. In addition, we were able to estimate the tracking error.

Let $D>0$ be given.

We consider continuous time processes $x(t)$ with a polynomial rate of growth, i.e., such that there exists $C>0$ and $c>0$ such that

$$
|x(t+\theta)| \leq C\left(|x(t)|+\theta^{c}\right), \quad t>0, \quad \theta>0 .
$$

In addition, we assume that there are intervals $[\alpha, \beta] \subset[0,+\infty)$ such that

$$
\sup _{t \in\left[t_{k}, t_{k+1}\right]}\left|x(t)-x\left(t_{k}\right)\right| \leq D \delta
$$

for all for all $k$ such that $\left[t_{k}, t_{k+1}\right] \subset[\alpha, \beta]$. In fact, $y(t)$ will be approaching $x(t)$ during these time intervals only. Therefore, a good approximation is not feasible if these intervals are too small or have too large gaps between them.

Remark 1 Clearly, (6) holds if $\left.x\right|_{[\alpha, \beta]}$ is absolutely continuous and $|d x(t) / d t| \leq D$. However, we prefer to use condition (6) since it is less restrictive; in particular, (6) holds for some discontinuous on $[\alpha, \beta]$ processes $x$.

Theorem 1 (i) Let $\tau=\inf \{m \in \mathcal{T}\}$. Then

$$
\tau \leq \inf \left\{m \geq 0: M_{0}\left(1+a+a^{2}+\cdots+a^{m}\right) \delta \geq\left|y_{0}-x(0)\right|+C\left(1+m^{c} \delta^{c}\right)\right\} .
$$

Here $C$ and $c$ are the constants from (6). 
(ii) Assume that $\bar{M} \geq 2 D$. Let $\theta=3 \log _{a}\left(M_{\tau} / \bar{M}\right)+6$. In this case, if (6) holds for all $k \in\{\tau, \tau+1, \tau+2, \ldots, \tau+\theta\}$, then there exists an integer $\eta \in\{\tau, \tau+1, \tau+2, \ldots, \tau+\theta\}$ such that

$$
M_{\eta}=\bar{M}, \quad\left|x\left(t_{\eta}\right)-y\left(t_{\eta}\right)\right| \leq(a \bar{M}+D) \delta .
$$

(iii) Assume that $\bar{M} \geq 2 D$, assume that (8) holds for some integer $\eta \geq 0$, and assume that (6) holds for $k \geq \eta$. Then $M_{k} \in\{\bar{M}, a \bar{M}\}$ for all $k \geq \eta$ and $M_{k}=\bar{M}$ for all $k \geq \eta$ such that $k \in \mathcal{T}$. In addition,

$$
\begin{array}{r}
\left|x\left(t_{k}\right)-y\left(t_{k}\right)\right| \leq(a \bar{M}+D) \delta \\
\sup _{t \in\left[t_{k}, t_{k+1}\right]}|x(t)-y(t)| \leq(a \bar{M}+2 D) \delta
\end{array}
$$

for all $k \geq \eta$.

The proof of the theorem is given in Appendix.

Let us discuss some implications of Theorem 1. As can be seen, $y(t)$ stats to approximate $x(t)$ after the time $t_{\eta}$ and until (6) is overstepped. The time period $\left[0, t_{\eta}\right]$ is used to bring the value $y(t)$ to a close proximity of $x(t)$. The time period $\left[t_{\tau}, t_{\eta}\right]$ is used to reduce the value $M_{k}$ from $M_{\tau}$ down to $\bar{M}$. The approximation error can be significant during the time interval $\left[0, t_{\eta}\right]$, if the distance $\left|y_{0}-x(0)\right|$ is large.

If a jump of $x$ occurs at time $s>t_{\eta}$, then Theorem 1 can be applied again for the initial time $t=s$ instead of $t=0$, and for $y_{0}$ and $M_{0}$ replaced by $y(\kappa \delta)$ and $M_{\kappa}$, where $\kappa=\min \{k: k \delta \geq s\}$.

If $\bar{M} \geq 2 D$, then, by Theorem 1 , the process $y(t)$ oscillates about the underlying process, and the error does not vanish even for constant $x(t)$. On the other hand, a choice of small $\bar{M}$ may lead to a larger time of proximity recovery after a jump of $x$.

Remark 2 The suggested algorithm is robust with respect to the errors caused by missed or misread signals $h_{k}$ for the models where the decoder is always aware that a signal was missed or misread, i.e., for the case of the so-called binary erasure channel. Obviously, there are models of channels with noise where these conditions are not satisfied. It could be interesting to find a way to modify an algorithm such that it will be robust with respect to the transmission errors for these models. 


\section{$3 \quad$ Illustrative examples}

In numerical experiments, we compared the performance of the Jayant's encoding algorithm [10] and modified version (1)-(4). We observed that, in all our experiments, the modified version (1)-(4) ensures faster recovering of the proximity after a jump of the underlying process. This is illustrated in Figures 1-2 presenting the results of the applications of the Jayant's encoding algorithm [10] and the suggested algorithm (1)-(4) of a discontinuous piecewise continuous process $x(t)$. Figures 1-2 show the process $x(t)$ and the corresponding processes $y(t)$ for $\delta=t_{k+1}-t_{k}=0.04$ and for $\delta=t_{k+1}-t_{k}=0.02$ respectively, $t \in[0,2]$. With these sampling rates, transmission of the encoded signals for $t \in[0,2]$ requires to transmit 50 bits only for $\delta=0.04$ and 100 bits only for $\delta=0.02$. For these examples, we used $y_{0}=5$, $a=1.5$, and $M_{0}=2 \delta=2 D$. The algorithm (1)-(4) was applied with $\bar{M}=2 \delta$. We used MATLAB for these calculations.

Figure 1: Example of a discontinuous input $x(t)$ and the corresponding estimate $y(t)$ for $\delta=0.04$ for Jayant's encoding algorithm [10] and for the suggested algorithm (1)-(4).

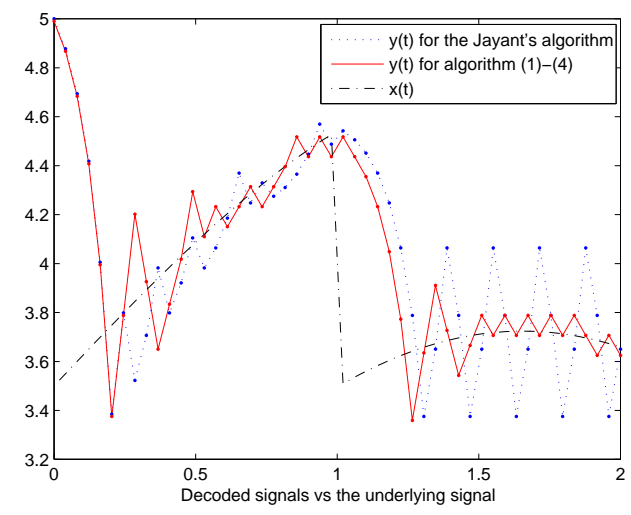

\section{Discussion and future developments}

1. The estimates in Theorem 1 represent the upper bounds for the worst case scenario; in practice, one should expect a better performance. A more informative estimate of the algorithm performance could be obtained in the stochastic setting, such as the mean square error given certain probabilistic characteristics of the input. In this setting, optimal selection of the parameter $a$ could be investigated. 
Figure 2: Example of a discontinuous input $x(t)$ and the corresponding estimate $y(t)$ for $\delta=0.02$, for Jayant's encoding algorithm [10] and for the suggested algorithm (1)-(4).

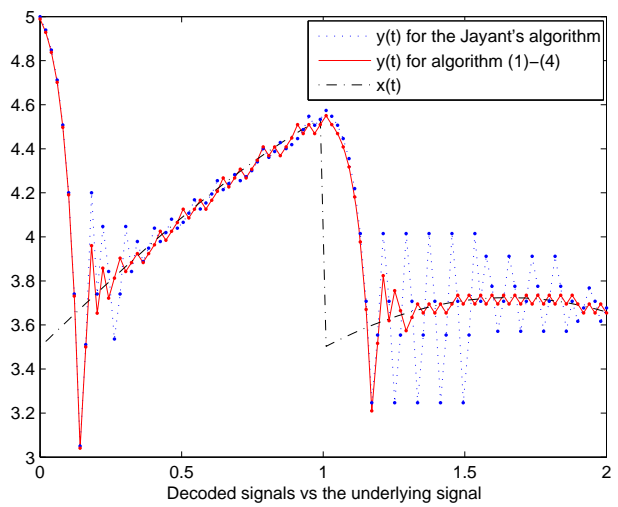

2. It could be interesting to extend the algorithm on vector processes $x(t)$.

3. The presented algorithm is causal, i.e., it collects current information and does not require the future values of the process. It could be interesting to estimate the loss of the effectiveness caused by the causality restrictions in comparison with the algorithms known in the rate-distortion theory in non-causal setting, where an entire signal $\left.x(t)\right|_{[0, T]}$ is known and has to be encoded, for some given interval [0,T] (see, e.g., [12], Ch. 13).

4. In theory, an arbitrarily close causal approximation in $L_{2}$-norm can be achieved by binomial processes with a fixed rate of change for general stochastic square integrable processes, including Ito and jump processes [13]. However, an algorithm of this approximation was not obtained therein. It could be interesting to investigate if the algorithm from the present paper can be used to achieve this kind of approximation.

\section{Appendix: Proof of Theorem 1}

To prove statement (i), it suffices to observe that

$$
\inf _{k \leq m}\left|y\left(t_{k}\right)-x\left(t_{k}\right)\right| \leq\left|y_{0}-x(0)\right|+C\left(1+m^{c} \delta^{c}\right)-M_{0}\left(1+a+a^{2}+\cdots+a^{m}\right) \delta .
$$

Let us prove statement (ii). For integer numbers $s \geq 0$, set

$$
\tau(0)=\tau=\inf \{m \in \mathcal{T}\}, \quad \tau(s)=\inf \{m>s: m \in \mathcal{T}\}, \quad s>0 .
$$


Let us prove first that, for any $s \in \mathcal{T}$,

$$
\tau(s) \leq s+3
$$

and

$$
\begin{array}{r}
\sup _{s \leq k \leq \tau(s)}\left|x\left(t_{k}\right)-y\left(t_{k}\right)\right| \leq\left(M_{s-1}+D\right) \delta, \\
\sup _{s \leq k \leq \tau(s)} \sup _{t \in\left[t_{k}, t_{k+1}\right]}|x(t)-y(t)| \leq\left(M_{s-1}+2 D\right) \delta .
\end{array}
$$

For certainty, we assume that $h_{s-1}=-1$. Since $s \in \mathcal{T}$, it follows that $h_{s}=1$ and $x\left(t_{s-1}\right) \in$ $\left[y_{s}, y_{s-1}\right]$. If $s+1 \in \mathcal{T}$ or $s+2 \in \mathcal{T}$ then (10) holds. Suppose that $s+1 \notin \mathcal{T}, s+2 \notin \mathcal{T}$, and $s+3 \notin \mathcal{T}$. Hence

$$
x\left(t_{s+3}\right) \in\left[y_{s}-4 D \delta, y_{s-1}+4 D \delta\right] .
$$

Since $s+1 \notin \mathcal{T}$ and $s+2 \notin \mathcal{T}$, it follows that $x\left(t_{s+3}\right) \in\left[y_{s-1}, y_{s-1}+4 D \delta\right)$. On the other hand,

$$
y\left(t_{s+3}\right)=y_{s-1}-M_{s-1} \delta+M_{s} \delta+M_{s+1} \delta+M_{s+2} \delta \geq y_{s-1}+2 M_{s} \delta \geq y_{s-1}+4 D \delta .
$$

It follows that $\{s+1, s+2, s+3\} \cap \mathcal{T} \neq \emptyset$ and (10) holds.

Let us prove (11). We have to consider the cases where $\tau(s)=s+1, s+2, s+3$ separately. Let us assume again that $h_{s-1}=-1$ and $h_{s}=1$.

We have that $M_{s-1} \geq 2 a D$ and $x\left(t_{s}\right) \in\left[y_{s-1}+D \delta, y_{s}\right]$.

Let us assume that $\tau(s)=s+1$. In this case,

$$
\begin{aligned}
& x\left(t_{s+1}\right) \leq y_{s+1}, \\
& x\left(t_{s+1}\right) \in\left[y_{s}-D \delta, y_{s+1}\right], \quad x\left(t_{s}\right) \in\left[y_{s}, y_{s+1}+D \delta\right], \\
& x(t) \in\left[y_{s}-D \delta, y_{s+1}+D \delta\right], \quad t \in\left[t_{s}, t_{s+1}\right], \\
& y(t)=y_{s}+M_{s}\left(t-t_{s}\right) \delta, \quad t \in\left[t_{s}, t_{s+1}\right] .
\end{aligned}
$$

Hence (11) holds for the case where $\tau(s)=s+1$.

Let us assume that $\tau(s)=s+2$. In this case,

$$
\begin{aligned}
& x\left(t_{s+2}\right) \leq y_{s+2}, \quad x\left(t_{s+1}\right)>y_{s+1}, \\
& x\left(t_{s+2}\right) \in\left[y_{s+1}-D \delta, y_{s+2}\right], \quad x\left(t_{s+1}\right) \in\left[y_{s+1}, y_{s+2}+D \delta\right], \\
& x\left(t_{s}\right) \in\left[y_{s+1}-D \delta, \min \left(y_{s-1}+D \delta, y_{s+2}+2 D\right],\right.
\end{aligned}
$$


and

$$
\begin{aligned}
& x(t) \in\left[y_{s+1}-D \delta, y_{s+2}+D \delta\right], \quad t \in\left[t_{s+1}, t_{s+2}\right], \\
& x(t) \in\left[y_{s+1}-D \delta, \min \left(y_{t-s}+2 D \delta, y_{s+2}+D \delta\right)\right], \quad t \in\left[t_{s}, t_{s+1}\right], \\
& y(t)=y_{s+i}+M_{s+i}\left(t-t_{s+i}\right) \delta, \quad t \in\left[t_{s+i}, t_{s+i+1}\right], \quad i=0,1,2 .
\end{aligned}
$$

Hence (11) holds for the case where $\tau(s)=s+2$.

Let us assume that $\tau(s)=s+3$. In this case,

$$
x\left(t_{s+3}\right) \leq y_{s+3}, \quad x\left(t_{s+2}\right)>y_{s+2}, \quad x\left(t_{s+1}\right)>y_{s+1},
$$

and

$$
\begin{aligned}
x\left(t_{s+3}\right) & \in\left[y_{s+2}-D \delta, y_{s+3}\right], \\
x\left(t_{s+2}\right) & \in\left[y_{s+2}, \min \left(y_{s-1}+3 D \delta, y_{s+3}+D \delta\right],\right. \\
x\left(t_{s+1}\right) & \in\left[\max \left(y_{s+1}, y_{s+2}-D \delta\right), \min \left(y_{s-1}+2 D \delta, y_{s+3}+2 D\right)\right], \\
x\left(t_{s}\right) & \in\left[y_{s+1}-D \delta, \min \left(y_{s-1}+D \delta, y_{s+2}+2 D\right)\right] .
\end{aligned}
$$

In addition,

$$
\begin{aligned}
& x(t) \in\left[y_{s+2}-D \delta, y_{s+3}+D \delta\right], \quad t \in\left[t_{s+2}, t_{s+3}\right], \\
& x(t) \in\left[y_{s+1}-D \delta, y_{s-1}+3 D \delta\right], \quad t \in\left[t_{s+1}, t_{s+2}\right], \\
& x(t) \in\left[y_{s+1}-D \delta, y_{s+2}+D \delta\right], \quad t \in\left[t_{s}, t_{s+1}\right], \\
& y(t)=y_{s+i}+M_{s+i}\left(t-t_{s+i}\right) \delta, \quad t \in\left[t_{s+i}, t_{s+i+1}\right], \quad i=0,1,2,3 .
\end{aligned}
$$

Hence (11) holds for the case where $\tau(s)=s+3$.

Let us prove that

$$
M_{\tau(s)} \leq \max \left(M_{s-1} / a, \bar{M}\right)
$$

We found above that $\rho=\tau(s) \leq s+3$. By the definitions, $M_{\rho}=\max \left(M_{\rho-1} / a, \bar{M}\right)$. Further, if $\rho=s+1$ then $M_{\rho}=\max \left(M_{s} / a, \bar{M}\right)=\max \left(\max \left(M_{s-1} / a, \bar{M}\right) / a, \bar{M}\right) \leq \max \left(M_{s-1} / a, \bar{M}\right)$. If $\rho=s+2$ then $M_{s+1}=M_{s}$ and $M_{\rho}=\max \left(M_{s+1} / a, \bar{M}\right)=\max \left(M_{s} / a, \bar{M}\right) \leq \max \left(M_{s-1} / a, \bar{M}\right)$, similarly to the previous case. If $\rho=s+3$ then $M_{s+1}=M_{s}=\max \left(M_{s-1} / a, \bar{M}\right), M_{s+2}=$ $a M_{s+1}=a M_{s}$, and $M_{\rho}=\max \left(M_{s+2} / a, \bar{M}\right)=\max \left(M_{s+1}, \bar{M}\right)=\max \left(M_{s}, \bar{M}\right)=\max \left(M_{s-1} / a, \bar{M}\right)$. Then (12) follows. 
Let us prove statement (ii). Let us define $\theta_{0}=\tau(0), \theta_{k}=\tau\left(\theta_{k-1}\right), k>0$. By (10) and (12) applied to $\theta_{k}$ instead of $\tau(0)$, it follows that $\theta_{k}-\theta_{k-1} \leq 3$ and $M_{\theta_{k}}=\max \left(M_{\theta_{k-1}-1} / a, \bar{M}\right)$. Hence $M_{\theta_{k}}=\max \left(a^{-k+1} M_{\tau(0)}, \bar{M}\right)$. It follows that $\eta$ exists and $\eta \leq \theta_{k}$, where $k \leq$ $\log _{a}\left(M_{\tau(0)} / \bar{M}\right)+2$ and $\theta_{k} \leq \tau(0)+3 k$.

Let us prove statement (iii). Let us observe that, in the sequence $\left(h_{\eta+1}, h_{\eta+2}, h_{\eta+3}, \ldots\right)$, there are no quadruple occurrences of the same symbol, i.e., for all $m \geq \eta$,

$$
\left(h_{m+1}, h_{m+2}, h_{m+3}, h_{m+4}\right) \neq \pm(1,1,1,1) .
$$

We will use the induction method. Assume that the statement holds for $k \in[\eta, m]$, where $m \in \mathcal{T}$. It suffices to show that there exists $m_{0} \in\{m+1, m+2, m+3\} \cap \mathcal{T}$ such that the statement holds for $k \in\left\{m+1, \ldots, m_{0}\right\}$. For certainty, we assume that $h_{m}=1$. This means that $M_{m}=\bar{M}$ and $h_{m-1}=-1$.

- Assume that $h_{m+1}=-1$. It follows that $M_{m+1}=\bar{M}$ and $m+1 \in \mathcal{T}$.

- Assume that $\left(h_{m+1}, h_{m+2}\right)=(1,-1)$. It follows that $\left(M_{m+1}, M_{m+2}\right)=(\bar{M}, \bar{M})$ and $m+2 \in \mathcal{T}$.

- Assume that $\left(h_{m+1}, h_{m+2}\right)=(1,1)$. It follows from (10) that $h_{m+3}=-1$. Hence $\left(M_{m+1}, M_{m+2}, M_{m+3}\right)=(\bar{M}, a \bar{M}, \bar{M})$ and $m+3 \in \mathcal{T}$.

In addition, (11) holds for $s=m$. By induction, the proof of (iii) follows. This completes the proof of Theorem 1.

\section{References}

[1] D.J. Stilwell, B.E. Bishop, "Platoons of underwater vehicles", IEEE Control Systems Magazine, vol. 20(6), pp. 45-52, 2000.

[2] W.S. Wong, R.W. Brockett, "Systems with finite communication bandwidth constraints - Part 1: state estimation problems", IEEE Trans. Automat. Contr., vol. 42 (9), pp. 1294-1299, 1997.

[3] G. Nair, R.J. Evans, "State estimation under bit-rate constraints", In Proc. 37th IEEE Conf. Decision and Control, 1998. 
[4] N.G Dokuchaev, A.V. Savkin, "A new class of hybrid dynamical systems: state estimators with bit-rate constraints", Intern. J. Hybrid Systems, vol. 1, No. 1, pp. 33-50, 2001.

[5] Z. Cvetković and I. Daubechies, "Single-bit oversampled A/D conversionwith exponential accurace in the bit-rate", IEEE Trans. Inf. Theory, vol. 53 (11), pp. 3979-3989, 2007.

[6] A. Kumar, P. Ishwar, and K.Ramchandran, "Dithered A/D Conversion of Smooth NonBandlimited Signals", IEEE Trans. Signal Process., vol. 58 (5), 2654-2656, 2010.

[7] E. Masry and S. Cambanis, Consistent estimation of continuous time signals from nonlinear transformations of noisy samples, IEEE Trans. Inf. Theory, vol. 27, pp. 84-96, Jan. 1981.

[8] E. Masry, "The reconstruction of analog signals from the sign of their noisy samples", IEEE Trans. Inf. Theory, vol. 27 (6), 735-745, 1981.

[9] A. Kumar and V.M. Prabhakaran, "Estimation of bandlimited signals from the signs of noisy samples". ICASSP 2013, Vancouver, BC, Canada, May 26-31, 5815-5819, 2013.

[10] N. S. Jayant, "Adaptive delta modulation with a one-bit memory", The Bell System Technical Journal, vol. 49, no. 3, pp. 321-342, 1970.

[11] S. Dandach, S. Dasgupta, B. Anderson, "Stability of adaptive delta modulators with forgetting factor and constant inputs", Intern. J. Adaptive Control and Signal Process., vol. 25 , pp. 723-739, 2011.

[12] T. M. Cover, J. A. Thomas, Elements of Information Theory, New York: Wiley, 2006.

[13] N. Dokuchaev, "On strong causal binomial approximation for stochastic processes", Discrete and Continuous Dynamical Systems - Series B (DCDS-B), vol. 19 (6), 1549 $1562,2014$. 